\title{
Euchromatic histone lysine methyltransferase 1 regulates cancer development in human gastric cancer by regulating E-cadherin
}

\author{
YAO YANG ${ }^{*}$, JIANFENG SHEN ${ }^{*}$, DONGYI YAN, BIAO YUAN, SHUN ZHANG, JING WEI and TAO DU \\ Department of Gastrointestinal Surgery, Shanghai East Hospital, \\ Tongji University School of Medicine, Shanghai 200120, P.R. China
}

Received February 20, 2017; Accepted February 27, 2018

DOI: $10.3892 / \mathrm{ol} .2018 .8506$

\begin{abstract}
Gastric cancer (GC) is among the most aggressive types of cancer and is the second leading cause of cancer-associated mortality worldwide. The specific role of deregulated expression/activity of histone methyltransferases (HMTs) in GC is poorly understood. The present study aimed to explore the possible oncogenic role of euchromatic histone lysine methyltransferase 1 (EHMT1) in gastric carcinogenesis. It was identified that EHMT1 was highly expressed in GC tissues compared with that in adjacent non-tumor tissues, and that EHMT1 expression levels were significantly associated with tumor stage and lymph node metastasis. Through knockdown of EHMT1 in the BGC-803 cell line, EHMT1 was demonstrated to promote a malignant phenotype, and to increase the wound healing, migration and invasion abilities of GC cells. Corresponding to these in vitro results, knockdown of EHMT1 also inhibited the peritoneal metastasis of GC cells in vivo. Furthermore, EHMT1 also regulated the expression of the epithelial-mesenchymal transition marker E-cadherin in vitro and in vivo. These results indicate that EHMT1 is upregulated in GC and serves an oncogenic role in GC development by regulating E-cadherin expression.
\end{abstract}

\section{Introduction}

Gastric cancer (GC), is among the most common types of cancer in Asia (1). The 5-year survival rate of patients with GC treated at an early stage is $90-95 \%$. However, if GC develops

Correspondence to: Dr Tao Du, Department of Gastrointestinal Surgery, Shanghai East Hospital, Tongji University School of Medicine, 150 Jimo Road, Shanghai 200120, P.R. China

E-mail: sfgidt1982@163.com

Abbreviations: GC, gastric cancer; HMTs, histone methyltransferases; EMT, epithelial-mesenchymal transition

${ }^{*}$ Contributed equally

Key words: euchromatic histone lysine methyltransferase 1, gastric cancer, migration, invasion, E-cadherin to a late stage, the survival rate is significantly decreased (2). Despite advances in the treatment of GC, a considerable number of patients remain with local recurrence or distant metastasis, and the underlying molecular mechanism of GC metastasis remains unclear (3). Therefore, investigation of the regulatory mechanisms underlying the activation of GC cell metastasis is required for the effective diagnosis and treatment of GC.

Deregulation of epigenetic mechanisms contributes to GC development and progression, including acetylation, methylation, phosphorylation and ubiquitination (4). Histone methylation has attracted increasing attention due to its participation in the process of heterochromatin formation, gene imprinting, $\mathrm{X}$ chromosome inactivation and gene transcriptional regulation $(5,6)$. Histone methyltransferases (HMTs) are a class of catalytic 1-3 ethyl group transfer to histone lysine or arginine, and are classified into histone lysine methyltransferases (HKMTs) and histone arginine methyltransferases (7). HKMTs can be further classified into 2 subgroups: SET domain and non-SET domain (8). Aberrant HMT expression has been identified in GC, and has been demonstrated to contribute to GC metastasis and development by promoting oncogene expression or inhibiting the expression of tumor suppressor genes. For example, depletion of EHMT2 inhibits cell growth and induces apoptosis in GC, indicating therapeutic potential in GC (9). High expression of SET domain containing (lysine methyltransferase) 8 (SET8) was associated with a shortened survival time in patients with GC, and the level of SET8 expression was identified as an independent predictor of GC outcome (10). Enhancer of zeste2 polycomb repressive complex 2 subunit can mediate the inhibition of S100A4 on E-cadherin, and regulate the proliferation and migration of GC cells (11). Abnormal EHMT1 expression in cancer tissues, including esophageal squamous cell cancer (12) and breast cancer (13), suggests that EHMT1 functions in tumor pathogenesis and progression. However, whether EHMT1 serves a role in GC development remains unknown. The present study aimed to elucidate the role of EHMT1 deregulation in GC carcinogenesis, to characterize its putative oncogenic role and its potential clinical impact.

\section{Materials and methods}

Tissues. The clinical research protocol of the present study was approved by the Ethical Committee of Shanghai East Hospital 
(Shanghai, China). GC tissues and matched non-tumor tissues were obtained from 97 patients who underwent curative surgery between March 2011 and September 2016 at the Department of Surgery, Shanghai East Hospital. None of the patients had received chemotherapy prior to surgery. Written informed consent was obtained from all participants. All tissue samples were immediately snap-frozen in liquid nitrogen or formalin-fixed $\left(4 \%\right.$ at $4^{\circ} \mathrm{C}$ for $\left.24 \mathrm{~h}\right)$ and paraffin-embedded.

Cell culture. The immortalized normal gastric epithelial cell line, GES-1, and the GC cell lines, BGC-803, AGS, KATO III and NCI-N87 (all cells were purchased from the library of the Chinese Academy of Sciences, Shanghai, China), were used in the present study. All cells were cultured in RPMI-1640 medium (Gibco; Thermo Fisher Scientific, Inc., Waltham, MA, USA) containing 10\% fetal calf serum (Gibco; Thermo Fisher Scientific, Inc.), $100 \mu \mathrm{g} / \mathrm{ml}$ streptomycin and $100 \mathrm{U} / \mathrm{ml}$ penicillin at $37^{\circ} \mathrm{C}$ in a humidified incubator containing $5 \% \mathrm{CO}_{2}$.

Xenograft model. All animal experiments were approved by the Experimental Animal Ethics Committee of Shanghai East Hospital and performed according to the Guide for the Institutional Animal Care and Use Committee of Shanghai Tongji University (Shanghai China). Specific pathogen-free grade, male, four-week-old, 10-12 g weight male BALB/c nude mice were purchased from the Institute of Zoology, Chinese Academy of Sciences (Beijing, China). Animals were housed in cages with wood chip beddings in a temperature-controlled room $\left(20-22^{\circ} \mathrm{C}\right)$ with a 12 -h light-dark cycle and $45-55 \%$ relative humidity, and were permitted free access to food and drinking water. In order to study the effect of EHMT1 on abdominal metastasis of gastric cancer and the expression of E-cadherin in the subcutaneous transplantation tumor, the mice were divided into 2 equal groups (five nude mice in each group), and subcutaneously $\left(6 \times 10^{5}\right.$ cells) or abdominally $\left(2 \times 10^{6}\right.$ cells $)$ injected with either BGC-803/NC or BGC-803/sh1-EHMT1 cells. All mice were sacrificed after 30 days. Subcutaneous tumor grafts were removed, fixed in $4 \%$ formalin for $24 \mathrm{~h}$ in $4^{\circ} \mathrm{C}$ to obtain 5- $\mu \mathrm{m}$ thick paraffin-embedded sections and analyzed by immunohistochemistry, and peritoneal metastasis nodules were counted and further analyzed. In the process, if the mice showed signs of cachexia or excessive ascites affecting their diet and activity, the experiment was promptly terminated.

Reverse transcription-quantitative polymerase chain reaction $(R T-q P C R)$. Total RNA from GC tissues and cell lines were extracted using TRIzol (Thermo Fisher Scientific, Inc.), according to the manufacturer's protocol. cDNA was synthesized from $1 \mu \mathrm{g}$ RNA using a reverse transcription kit (Promega Corporation, Madison, WI, USA), according to the manufacturer's protocols. RT-qPCR was performed using SYBR-Green PCR Master mix (Applied Biosystems; Thermo Fisher Scientific, Inc.), according to the manufacturer's instructions. GAPDH was used as an internal control. The PCR primers were as follows: EHMT1 forward, 5'-CAT GCAGCCAGTAAAGATCCC-3', and reverse, 5'-CTGCTG TCGTCCAAAGTCAG-3'; and GAPDH forward, 5'-TTGGCA TCGTTGAGGGTCT-3' and reverse, 5'-CAGTGGGAACAC
GGAAAGC-3'. PCR reactions were performed with an initial denaturation at $95^{\circ} \mathrm{C}$ for $5 \mathrm{~min}$, followed by 30 cycles of $95^{\circ} \mathrm{C}$ for $30 \mathrm{sec}, 57^{\circ} \mathrm{C}$ for $30 \mathrm{sec}$ and $72^{\circ} \mathrm{C}$ for $30 \mathrm{sec}$, with a final extension at $72^{\circ} \mathrm{C}$ for $10 \mathrm{~min}$. Gene expression was quantified using the $2^{-\Delta \Delta \mathrm{Cq}}$ method (14).

Immunohistochemistry (IHC). Sections of a 5- $\mu$ m thickness were sliced from the paraffin-embedded tissues of the mice or patients, and deparaffinized (100\% xylene) and rehydrated in an ethanol series (100-50\%). The sections were then treated in $0.01 \mathrm{~mol} / 1$ citrate buffer ( $\mathrm{pH} \mathrm{6.0)}$ for antigen retrieval. Endogenous peroxidase activity was inhibited following incubation with methanol containing $0.3 \% \mathrm{H}_{2} \mathrm{O}_{2}$ for $30 \mathrm{~min}$. Subsequently, sections were incubated with antibodies detecting EHMT1 (cat. no. ab41969; 1:200; Abcam, Cambridge, UK) and E-cadherin (cat. no. ab76055; 1:200; Abcam) primary antibodies at $37^{\circ} \mathrm{C}$ for $2 \mathrm{~h}$. Normal IgG (cat. no. ab6728; 1:200; Abcam) was used as a negative control. The slides were washed with PBS and incubated for $2 \mathrm{~h}$ at room temperature with an EnVision kit (cat. no. GK500705; Dako; Agilent Technologies, Inc., Santa Clara, CA, USA), according to the manufacturer's protocol. The percentage of positive tumor cells was scored as follows: $<10 \%$, score $0 ; 10-25 \%$, score $1 ; 26-50 \%$, score 2 ; $51-75 \%$, score 3 ; and $>75 \%$, score 4 . Intensity of staining was qualitatively evaluated as follows: Negative, score 0 ; weak, score 1; moderate, score 2; or strong, score 3. The percentage score was multiplied by the intensity score to give a final staining score. Final scores of $0-4$ were considered to indicate weak expression, whereas final scores of 4-12 were considered to indicate strong expression.

Western blot analysis. Whole cell proteins were extracted from cells using radioimmunoprecipitation buffer containing a Protease Inhibitor Cocktail (Pierce; Thermo Fisher Scientific, Inc.). Protein was quantified using a bicinchoninic Protein Assay kit (Pierce; Thermo Fisher Scientific, Inc.). Cell extracts $(50 \mu \mathrm{g})$ were separated by $10 \%$ SDS-PAGE, and transferred onto polyvinylidene fluoride membranes. Membranes were blocked with $5 \%$ nonfat milk in Tris-buffered saline (TBS) for $2 \mathrm{~h}$ at $25^{\circ} \mathrm{C}$ and incubated with primary antibodies at $4^{\circ} \mathrm{C}$ overnight. The primary antibodies used were mouse anti-EHMT1 (cat. no. ab41969; 1:1,000), mouse anti-Ecadherin (cat. no. ab76055; 1:1,000) and mouse anti-GAPDH (cat. no. ab8245; 1:1,000; all Abcam). Membranes were then washed three times in 1xTBS-Tween solution for $15 \mathrm{~min}$, and incubated with anti-mouse IgG (horseradish peroxidase conjugated) secondary antibodies for $2 \mathrm{~h}$ in $25^{\circ} \mathrm{C}$ (cat. no. ab193651; 1:5,000; Abcam). Signals were visualized using an enhanced chemiluminescence kit (cat. no. WBKLS0050; EMD Millipore, Billerica, MA, USA).

Plasmid construction and transfection. EHMT1 short hairpin RNAs (shRNAs) and negative control (NC) were obtained from OBIO (Shanghai, China). EHMT1 sh1RNA (targeting sequence, 5'-CGAGTCAATAACGCCAGCTAT-3'), sh2RNA (targeting sequence, 5'-CCTCGGTTCTGAGTCGTATAA-3') or NC (targeting sequence, 5'-TTCTCCGAACGTGTC ACGT-3') were cloned into G418 plasmids (Gibco; Thermo Fisher Scientific, Inc.). The plasmid products were then transfected separately into BGC-803 cells $\left(6 \times 10^{4}\right.$ cells/well $)$ 

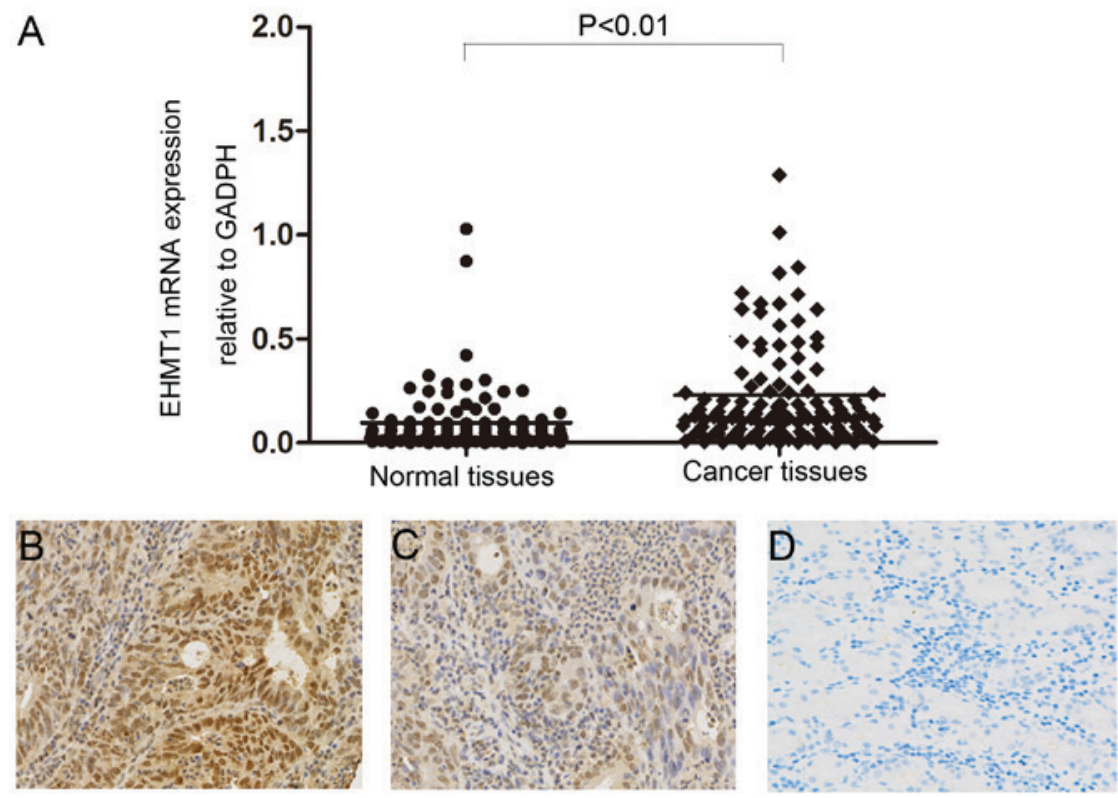

Figure 1. Expression of EHMT1 in GC tissues. (A) EHMT1 mRNA expression in GC tissues and paired adjacent non-tumor tissues was analyzed by reverse transcription-quantitative polymerase chain reaction. Data are presented as $2^{-\Delta \Delta C q}$ values. Immunohistochemical analysis of EHMT1 expression in GC tissues classified as (B) strong-positive or (C) weak-positive. (D) Negative EHMT1 expression in adjacent normal tissue. Magnification, x200. EHMT1, euchromatic histone lysine methyltransferase 1 ; GC, gastric cancer.

at $1,500 \mu \mathrm{g} / \mathrm{ml}$ using Lipofectamine 2000 (Thermo Fisher Scientific, Inc.), according to the manufacturer's protocol. A total of one month later following transfection, stably transfected cells were detected using RT-qPCR and western blotting.

Wound healing assay. BGC-803/sh1, BGC-803/sh2 cells and NC cells were cultured as a monolayer to $100 \%$ confluence in 6-well plates. The cells were then scratched with a sterile pipette tip. The plates were washed with PBS to remove the cellular debris, and then cultured in RPMI-1640 serum-free medium (Gibco; Thermo Fisher Scientific, Inc.). The extent of wound healing was observed at 0 and $48 \mathrm{~h}$ under an inverted phase contrast microscope.

Migration and invasion assays. The cell migration and invasion abilities were measured using a 24-well Transwell chamber with $8-\mu \mathrm{m}$ pore inserts (Corning Inc., Corning, NY, USA). For the migration assay, $1.0 \times 10^{5}$ cells in $200 \mu 1$ RPMI-1640 serumfree medium (Gibco; Thermo Fisher Scientific, Inc.) were placed in the upper chamber, whereas $600 \mu \mathrm{l}$ media with $10 \%$ FBS was placed in the lower chamber. After $24 \mathrm{~h}$, cells remaining on the upper side of inserts were gently scraped off and cells on the lower surface were fixed in $100 \%$ methanol and stained with $0.5 \%$ crystal violet $\left(37^{\circ} \mathrm{C}\right.$ for $30 \mathrm{~min}$ ). The invasion assay was performed according to the same protocol as the migration assay; however, the upper chamber was pre-coated with Matrigel. For the two assays, the stained cells were counted in 5 randomly selected fields under an inverted light microscope.

Statistical analysis. The association between EHMT1 expression and clinicopathological characteristics was analyzed using Pearson's $\chi^{2}$ test. The differences between 2 groups were analyzed using Student's t-test, and the differences between $\geq 3$ groups were analyzed using one-way analysis of variance with Bonferroni's post-hoc test. All statistical analyses were performed using SPSS 18.0 software (SPSS Inc., Chicago, IL, USA). $\mathrm{P}<0.05$ was considered to indicate a statistically significant difference.

\section{Results}

EHMT1 is overexpressed in GC tissues and is associated with tumor stage and lymph node metastasis. RT-qPCR was performed to analyze mRNA expression of EHMT1 in GC tumor tissues and matched non-tumor tissues. The mRNA level of EHMT1 in GC tissues was significantly increased compared with that of adjacent non-tumor tissues $(\mathrm{P}<0.01$; Fig. 1A). The protein expression level of EHMT1 in gastric tissues was analyzed by IHC, revealing increased EHMT1 staining in GC tissues compared with that in non-tumor tissues (Fig. 1B-D). The association between EHMT1 protein expression and the clinicopathological characteristics of 97 patients with GC are presented in Table I. $\chi^{2}$ analysis suggested that high expression of EHMT1 in GC was significantly associated with tumor stage $(\mathrm{P}=0.033)$ and lymph node metastasis $(\mathrm{P}=0.003)$. However, there was no statistically significant association between EHMT1 expression and other clinicopathological features, including sex, age and tumor size.

Construction of stable EHMT1-knockdown GC cell lines. EHMT1 expression was investigated in the immortalized gastric epithelial cell line, GES-1, and in a series of GC cell lines, including BGC-803, NCI-N87, AGS and KATO-III. Among these cell lines, BGC-803, NCI-N87, AGS and KATO-III exhibited increased EHMT1 expression compared with GES-1 ( $\mathrm{P}<0.01$; Fig. $2 \mathrm{~A}$ and $\mathrm{B})$. To further explore the functions of EHMT1 in GC cells, knockdown of EHMT1 
Table I. Association between EHMT1 expression and clinicopathological factors of gastric cancer patients.

EHMT1 protein expression

\begin{tabular}{|c|c|c|c|c|}
\hline & & & & \\
\hline Clinicopathological factor & $\mathrm{n}$ & Positive $(\mathrm{n}=65)$ & Negative $(n=32)$ & P-value \\
\hline Sex & & & & 0.420 \\
\hline Male & 74 & 48 & 26 & \\
\hline Female & 23 & 17 & 6 & \\
\hline Age, years & & & & 0.497 \\
\hline$\geq 60$ & 59 & 38 & 21 & \\
\hline$<60$ & 38 & 27 & 11 & \\
\hline Tumor differentiation & & & & 0.517 \\
\hline Well to moderate & 38 & 24 & 14 & \\
\hline Poor & 59 & 41 & 18 & \\
\hline Tumor location & & & & 0.295 \\
\hline Gastric fundus & 3 & 1 & 2 & \\
\hline Gastric corpus & 41 & 30 & 11 & \\
\hline Pylorus & 53 & 34 & 19 & \\
\hline Tumor size, $\mathrm{cm}$ & & & & 0.430 \\
\hline$\leq 3$ & 46 & 29 & 17 & \\
\hline$>3$ & 51 & 36 & 15 & \\
\hline Tumor stage & & & & 0.033 \\
\hline $\mathrm{T} 1-\mathrm{T} 2$ & 37 & 20 & 17 & \\
\hline $\mathrm{T} 3-\mathrm{T} 4$ & 60 & 45 & 15 & \\
\hline Lymph node metastasis & & & & 0.003 \\
\hline Negative & 43 & 22 & 21 & \\
\hline Positive & 54 & 43 & 11 & \\
\hline Distant metastasis & & & & 0.316 \\
\hline Negative & 95 & 63 & 32 & \\
\hline Positive & 2 & 2 & 0 & \\
\hline TNM stage & & & & 0.837 \\
\hline $\mathrm{I}+\mathrm{II}$ & 38 & 25 & 13 & \\
\hline III+IV & 59 & 40 & 19 & \\
\hline
\end{tabular}

Positive EHMT1 expression includes all positive cases, including weak and strong staining. EHMT1, euchromatic histone lysine methyltransferase 1; TNM, tumor-node-metastasis.

expression was performed in BGC-803 cell lines, and experimentally validated $(\mathrm{P}<0.01$; Fig. $2 \mathrm{C}$ and $\mathrm{D})$.

EHMT1 promotes wound healing, migration and invasion of $G C$ cells. Wound healing assays were performed to investigate the effect of EHMT1 on GC cell motility. It was observed that the distance between wound edges in the BGC-803/sh1-EHMT1 and BGC-803/sh2-EHMT1 cells was large compared with that in the control cells $(\mathrm{P}<0.01$; Fig. 3A and B).

The effect of EHMT1 on GC cell migration and invasion, which are key determinants of malignant progression and metastasis, was also assessed using Transwell assays. After $24 \mathrm{~h}$ of incubation, cells were counted under an inverted microscope. As illustrated in Fig. 3C and D, the number of cells that migrated into the lower chamber was significantly lower in BGC-803/sh1-EHMT1 and BGC-803/sh2-EHMT1 cells compared with that in BGC-803/NC cells in the migration and invasion assays (all $\mathrm{P}<0.01$ ).

EHMT1 expression promotes peritoneal metastasis of GC cells. Based on the aforementioned in vitro results, the in vivo function of overexpressed EHMT1 was investigated by abdominally injecting BGC-803/sh1-EHMT1 and negative control cells into nude mice. A total of 30 days following injection, the mice were sacrificed and the peritoneal nodules were evaluated. As demonstrated in Fig. 4A, increased peritoneal spread was observed in the negative control group compared with that in the BGC-803/sh1-EHMT1 group. Following counting of the nodules, it was evident that there were significantly fewer peritoneal nodules in mice injected with the BGC-803/sh1-EHMT1 cells compared with the number in mice injected with negative control cells $(\mathrm{P}<0.01$; Fig. 4B). The xenograft model experiment was, therefore, consistent 
A

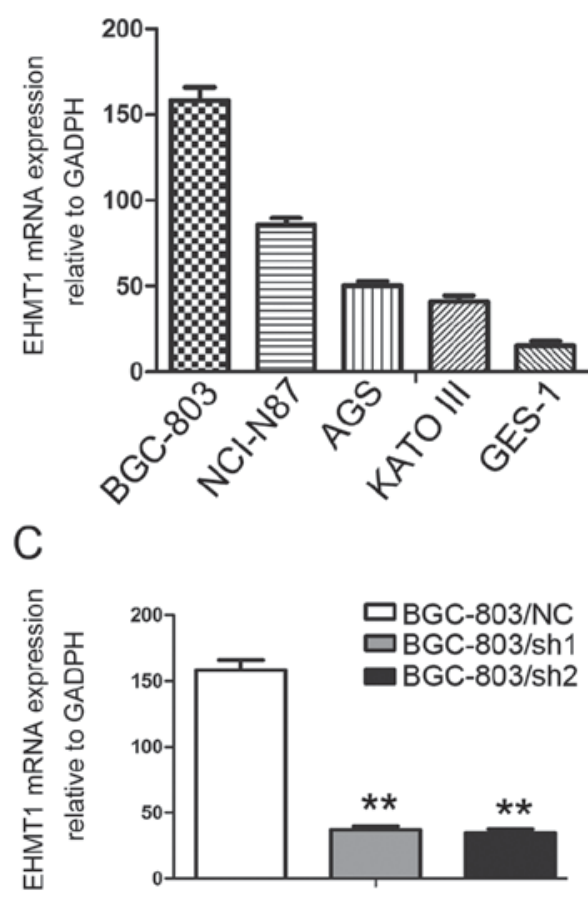

B

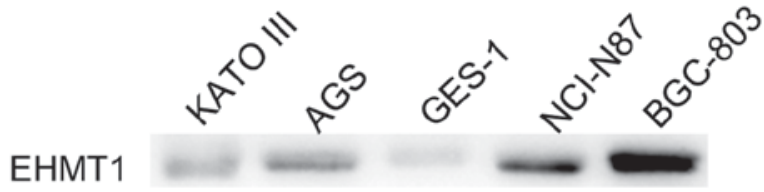

$\mathrm{GAPDH}=0$

D

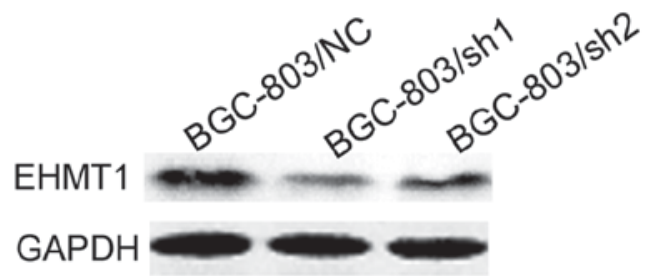

Figure 2. Expression of EHMT1 in GC cell lines. (A) EHMT1 mRNA expression in GC cells analyzed by RT-qPCR. (B) EHMT1 protein expression in GC cells analyzed by western blotting methods. EHMT1 (C) mRNA and (D) protein expression were investigated by RT-qPCR and western blotting following BGC-803 transfection. ${ }^{* *} \mathrm{P}<0.01$ vs. control. EHMT1, euchromatic histone lysine methyltransferase 1; GC, gastric cancer; RT-qPCR, reverse transcription-quantitative polymerase chain reaction; sh1, EHMT1 short hairpin RNA 1; sh2, EHMT1 short hairpin RNA 2; NC, negative control.

with the in vitro results, confirming that EHMT1 expression in GC promotes GC metastasis.

EHMT1 promotes GC invasion and metastasis by silencing E-cadherin. E-cadherin is a marker of tumor metastasis, and E-cadherin protein expression was upregulated in BGC-803/sh1-EHMT1 cells compared with that in BGC-803/NC cells (Fig. 4C). To further verify the effect of EHMT1 on E-cadherin, BGC-803/sh1-EHMT1 and BGC-803/NC cells were subcutaneously injected into BALB/c nude mice. The maximum tumor volume of xenografts derived from the BGC-803/sh1-EHMT1 and BGC-803/NC groups was 0.991 and $0.861 \mathrm{~cm}^{3}$, respectively. In addition, the maximum weight of the tumors derived from the BGC-803/sh1-EHMT1 and BGC-803/NC groups was 0.661 and $0.574 \mathrm{~g}$, respectively. IHC suggested that EHMT1 silenced E-cadherin expression in subcutaneous tumors, thus promoting tumor metastasis (Fig. 4D).

\section{Discussion}

Histone modifications, including ubiquitination, phosphorylation, acetylation and methylation serve critical roles in transcriptional repression and activation through the regulation of chromatin structure (15). EHMT1 has been reported to be upregulated in esophageal squamous cell cancer (12) and breast cancer (13). However, to the best of our knowledge, the expression level of EHMT1 in GC remains unknown. In the present study, the significant upregulation of EHMT1 in GC was demonstrated using RT-qPCR and IHC, and its expression was associated with lymph node metastasis and tumor stage. It was also demonstrated that EHMT1 promotes the metastasis ability of GC cells and suppresses E-cadherin expression, which may contribute to the existing understanding of GC development.

The transcriptional activity of a gene is often unaffected by DNA methylation, but is determined by chromatinstate (16). Methylated histones allow close DNA packing and a dense chromatin structure, resulting in a decrease in the transcriptional activity of the gene in question (13). Fritsch et al established that EHMT1 can methylate the histone and promoter of specific genes, leading to decreased transcriptional activity (17). EHMT1 serves an important role in the methylation and dimethylation of histone H3 lysine 9 (H3K9) euchromatin, activating a series of downstream reactions and producing corresponding physiological functions. In the majority of cases, EHMT1 will first form a heterodimer with G9a (18). The G9a/EHMT1 complex can inhibit the expression of p53 by demethylating K373 at the C-terminus of the p53 gene, indicating a promotive effect of EHMT1 in cancer (19). In the Mage-a tumor stem cell line, H3K9 demethylation (H3K9me2) mediated by the EHMT1/G9a complex has an inhibitory effect on the expression of its antigenic gene (target of tumor vaccine currently undergoing clinical evaluation world-wide) (20). The surface antigens of tumor cells are targets of therapeutic drugs (21), and the EHMT1/G9a complex may function in immune avoidance and therapeutic resistance effects. Epithelial-mesenchymal transition (EMT) refers to the biological process by which epithelial cells are transformed into mesenchymal cells, and is considered to 


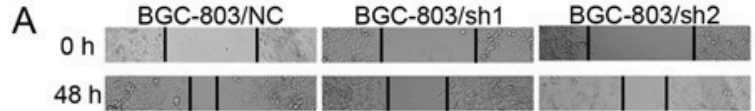

B

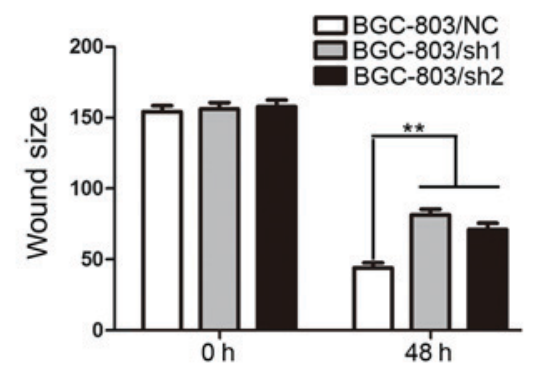

C

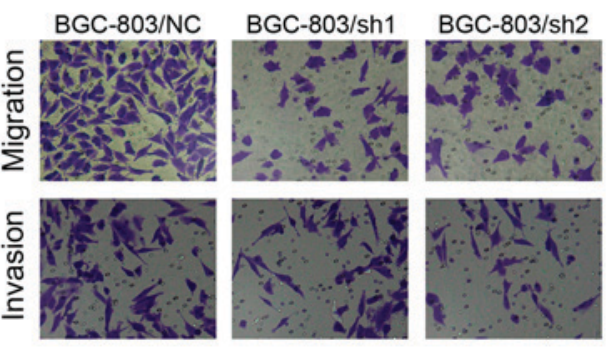

D

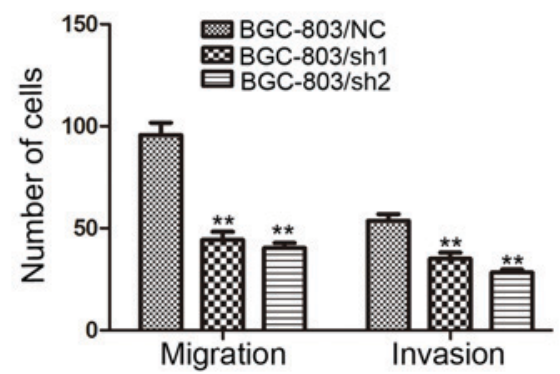

Figure 3. EHMT1 promotes the migration and invasion of GC cells (A) BGC-803 cells were transfected with EHMT1 shRNA1/2 or NC shRNA, and representative images of wound healing assays were captured at 0 and $48 \mathrm{~h}$ post-wounding (magnification, $\mathrm{x} 200$ ). (B) Quantification of the distance between wound edges of BGC-803/NC, BGC-803/sh1 and BGC-803/sh2 cells. (C) Representative images of migratory and invasive cells that traversed the micropore membrane following transfection with crystal violet staining (magnification, x200). (D) Average number of migratory cells and invasive cells from 5 fields of view. Data are represented as the mean \pm standard deviation of 3 independent experiments. ${ }^{* *} \mathrm{P}<0.01$ vs. control. EHMT1, euchromatic histone lysine methyltransferase 1; GC, gastric cancer; shRNA, short hairpin RNA; NC, negative control; sh1, EHMT1 shRNA 1; sh2, EHMT1 shRNA 2.

be an important marker of tumor progression. The loss of E-cadherin expression is indicative of the migration and invasion of malignant tumor cells (22-24). Previous studies have demonstrated that G9a forms a complex with Snail and binds to the E-cadherin promoter, resulting in $\mathrm{H} 3 \mathrm{~K} 9 \mathrm{me} 2$ activity and increased potential for EMT and metastasis $(25,26)$. Considering that EHMT1 and G9a exist as dimers, the present study investigated whether EHMT1 can affect the expression of E-cadherin. As expected, EHMT1 inhibited the expression of E-cadherin in vitro and in vivo. Thus, EHMT1 may promote the process of GC metastasis by influencing EMT in GC cells.

In conclusion, the present study demonstrated that EHMT1 was highly expressed in GC, and was associated with lymph node metastasis and tumor stage. Depletion of EHMT1 expression inhibited GC cell wound healing, migration and invasion. These results highlight the potential of EHMT1 as a potential therapeutic target for GC.
A

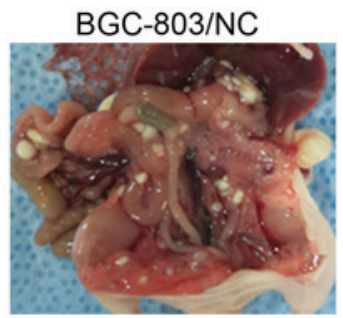

B
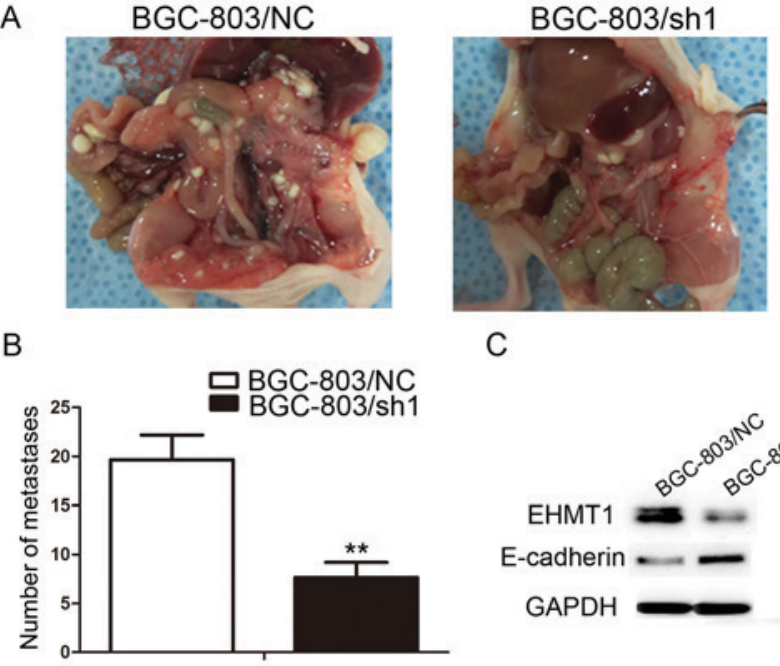

C

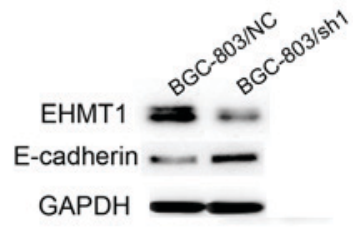

D
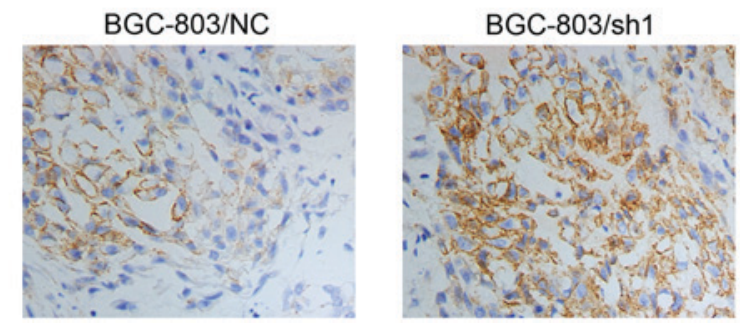

Figure 4. EHMT1 enhances peritoneal spreading of GC cells in vivo and regulates expression of E-cadherin in GC cell lines and subcutaneous tumors. (A) Representative images of peritoneal spreading and metastasis of BGC-803/NC and BGC-803/sh1 cell xenografts. (B) Quantitative analysis of metastases in each group. (C) Western blotting of E-cadherin in the BGC-803/sh1 and BGC-803/NC groups, with GAPDH used as a loading control. (D) The expression of E-cadherin in the subcutaneous tumor grafts analyzed by immunohistochemistry (magnification, x200). EHMT1, euchromatic histone lysine methyltransferase 1; GC, gastric cancer; NC, negative control; sh1, EHMT1 short hairpin RNA 1; sh2, EHMT1 short hairpin RNA 2.

\section{Acknowledgements}

Not applicable.

\section{Funding}

The present study was supported by grants from the Science and Technology Development Fund of Pudong New District (grant no. PKJ2016-Y60) and the Young Talent program of Tongji University (grant no. 1507219045).

\section{Availability of data and materials}

All data generated or analyzed during this study are included in this published article. .

\section{Authors' contributions}

TD and YY designed the research. YY, JFS and DY were responsible for the implementation of the experiment and acquisition of data. BY, SZ and JW analysed and interpreted the data. TD, YY and JFS wrote, reviewed and revised the manuscript. The final version of the manuscript has been read and approved by all authors. 


\section{Ethics approval and consent to participate}

Experiments using human tissues were approved by the Ethical Committee of Shanghai East Hospital (Shanghai, China), and written informed consent was obtained from all participants. All animal experiments were approved by the Experimental Animal Ethics Committee of Shanghai East Hospital and performed according to the Guide for the Institutional Animal Care and Use Committee of Shanghai Tongji University (Shanghai, China).

\section{Consent for publication}

All patients provided written informed consent for the publication of data.

\section{Competing interests}

The authors declare that they have no competing interests.

\section{References}

1. Hartgrink HH, Jansen EP, van Grieken NC and van de Velde CJ: Gastric cancer. Lancet 374: 477-490, 2009.

2. Correa P: Gastric cancer: Overview. Gastroenterol Clin North Am 42: 211-217, 2013.

3. Hanahan D and Weinberg RA: Hallmarks of cancer: The next generation. Cell 144: 646-674, 2011.

4. Patel TN, Roy S and Ravi R: Gastric cancer and related epigenetic alterations. Ecancermedicalscience 11: 714, 2017.

5. Huang L and Xu AM: SET and MYND domain containing protein 3 in cancer. Am J Transl Res 9: 1-14, 2017.

6. Greer EL and Shi Y: Histone methylation: A dynamic mark in health, disease and inheritance. Nat Rev Genet 13: 343-357, 2012

7. Albert $\mathrm{M}$ and Helin $\mathrm{K}$ : Histone methyltransferases in cancer. Semin Cell Dev Biol 21: 209-220, 2010.

8. Wood A and Shilatifard A: Posttranslational modifications of histones by methylation. Adv Protein Chem 67: 201-222, 2004.

9. Lin X, Huang Y, Zou Y, Chen X and Ma X: Depletion of G9a gene induces cell apoptosis in human gastric carcinoma. Oncol Rep 35: 3041-3049, 2016.

10. Shi XL, Guo ZJ, Wang XL, Liu XL and Shi GF: SET8 expression is associated with overall survival in gastric cancer. Genet Mol Res 14: 15609-15615, 2015.

11. Liu S, Chen D, Shen W, Chen L, Yu A, Fu H, Sun K and Sun X: EZH2 mediates the regulation of S100A4 on E-cadherin expression and the proliferation, migration of gastric cancer cells Hepatogastroenterology 62: 737-741, 2015.
12. Guan X, Zhong X, Men W, Gong S, Zhang L and Han Y: Analysis of EHMT1 expression and its correlations with clinical significance in esophageal squamous cell cancer. Mol Clin Oncol 2: 76-80, 2014.

13. Cebrian A,Pharoah PD, Ahmed S, Ropero S, Fraga MF, Smith PL, Conroy D, Luben R, Perkins B, Easton DF, et al: Genetic variants in epigenetic genes and breast cancer risk. Carcinogenesis 27: 1661-1669, 2006.

14. Livak KJ and Schmittgen TD: Analysis of relative gene expression data using real-time quantitative PCR and the 2(-Delta Delta C(T)) method. Methods 25:402-408, 2001.

15. Peterson CL and Laniel MA: Histones and histone modifications. Curr Biol 14: R546-R551, 2004.

16. Ordway JM and Curran T: Methylation matters: Modeling a manageable genome. Cell Growth Differ 13: 149-162, 2002.

17. Fritsch L, Robin P, Mathieu JR, Souidi M, Hinaux H, Rougeulle C, Harel-Bellan A, Ameyar-Zazoua M and Ait-Si-Ali S: A subset of the histone H3 lysine 9 methyltransferases Suv39h1, G9a, GLP, and SETDB1 participate in a multimeric complex. Mol Cell 37: 46-56, 2010.

18. Xiong Y, Li F, Babault N, Dong A, Zeng H, Wu H, Chen X Arrowsmith CH, Brown PJ, Liu J, et al: Discovery of potent and selective inhibitors for G9a-Like protein (GLP) lysine methyltransferase. J Med Chem 60: 1876-1891, 2017.

19. Huang J, Dorsey J, Chuikov S, Pérez-Burgos L, Zhang X, Jenuwein T, Reinberg D and Berger SL: G9a and Glp methylate lysine 373 in the tumor suppressor p53. J Biol Chem 285: 9636-9641, 2010

20. Link PA, Gangisetty O, James SR, Woloszynska-Read A, Tachibana M, Shinkai Y and Karpf AR: Distinct roles for histone methyltransferases G9a and GLP in cancer germ-line antigen gene regulation in human cancer cells and murine embryonic stem cells. Mol Cancer Res 7: 851-862, 2009.

21. Simpson AJ, Caballero OL, Jungbluth A, Chen YT and Old LJ: Cancer/testis antigens, gametogenesis and cancer. Nat Rev Cancer 5: 615-625, 2005.

22. Biddle A and Mackenzie IC: Cancer stem cells and EMT in carcinoma. Cancer metastasis Rev, 2012 (Epub Ahead of Print).

23. Yilmaz M and Christofori G: EMT, the cytoskeleton, and cancer cell invasion. Cancer Metastasis Rev 28: 15-33, 2009.

24. Huang L, Wu RL and Xu AM: Epithelial-mesenchymal transition in gastric cancer. Am J Transl Res 7: 2141-2158, 2015.

25. Liu S, Ye D, Guo W, Yu W, He Y, Hu J, Wang Y, Zhang L, Liao Y, Song H, et al: G9a is essential for EMT-mediated metastasis and maintenance of cancer stem cell-like characters in head and neck squamous cell carcinoma. Oncotarget 6: 6887-6901, 2015.

26. Dong C, Wu Y, Yao J, Wang Y, Yu Y, Rychahou PG, Evers BM and Zhou BP: G9a interacts with Snail and is critical for Snail-mediated E-cadherin repression in human breast cancer. J Clin Invest 122: 1469-1486, 2012. 